\title{
Come parlano gli italiani ${ }^{1}$
}

\section{Tullio De Mauro}

Università «La Sapienza», Roma

\begin{abstract}
In molti paesi del mondo la realtà plurilingue delle società è stata a lungo soffocata o ignorata. Dalla seconda metà del Novecento in molti, forse in tutti i paesi, un intreccio di fenomeni sociopolitici ed economici e il progresso degli studi linguistici hanno portato alla ribalta le effettive realtà plurilingui. L'Italia è, dai secoli del Medio evo, un paese accentuatamente plurilingue. I deficit di istruzione hanno a lungo reso difficile la convergenza verso un idioma unico, cioè verso il fiorentino considerato dal ceto colto, fin dal Cinquecento, come la lingua nazionale..La convergenza si è finalmente realizzata, sotto la spinta di fattori demografici, economici e, in parte, educativi soltanto in anni recenti. L'adozione dell'italiano coinvolge ormai oltre il $95 \%$ della popolazione, che, tuttavia, al $60 \%$ conserva l'uso di uno dei molti dialetti caratteristici del Paese o di una delle 14 lingue di minoranza. L'uso della lingua comune è tuttora segnato sia da tratti oggettivamente contrastanti o sovrabbondanti nella morfologia e nel lessico sia da notevoli variazioni regionali sia, infine, da difficoltà che i locutori incontrano nel maneggiare una lingua di antica tradizione e non semplice.
\end{abstract}

Parole chiave: italiano, dialetti italiani, plurilinguismo, alfabetizzazione.

\section{Abstract}

In many countries around the world, multilingual reality has long been suppressed or ignored. From the second half of the twentieth century in many if not in all countries, an interweaving of socio-political and economic phenomena and the progress of language studies have brought multilingual realities into the limelight. From the centuries of the Middle Ages, Italy has been an emphatically multilingual country. The deficits in education have long hindered the convergence towards a single language, that is to say towards the cautious Florentine from the cultured class, at the end of the Sixteenth century, as the national language. The convergence itself was finally achieved only in recent years under the impetus of demographic, economic and, in part, educational factors. Now, more than $90 \%$ of the population have adopted Italian, although $60 \%$ of them preserve the use of one of the many characteristic dialects of the country or one of the 14 minority languages. The use of the common tongue is still marked both by objectively clashing or overabun-

1. Rielaborazione del testo di una conferenza tenuta il 22 marzo 2004 all'Università di Barcellona. 
dant features in the morphology and vocabulary, by considerable regional variations and, finally, by the difficulty that speakers must face in dealing with an ancient, traditional and far from easy language.

Key words: Italian, Italian dialects, multilingualism, literacy.

1.

L'Italia d'oggi, guardata di lontano, con uno sguardo che l'abbracci nell'insieme e a confronto con gli altri paesi del mondo, è uno dei tanti paesi segnati da un alto grado di plurilinguismo. Dappertutto e anche, ovviamente, nell'Italia d'oggi ciò che diciamo "plurilinguismo" si atteggia in due differenti modi, intrecciati ma non identici. Vi è quel primo modo di presentarsi del plurilinguismo che già Dante metteva in evidenza nella pagina iniziale del De vulgari eloquentia:

Vulgarem locutionem appellamus eam quam infantes assuefiunt ab assistentibus cum primitus distinguere voces incipiunt, vel, quod brevius dici potest, vulgarem locutionem asserimus quam sine omni regula nutricem imitantes accipimus. Est et inde alia locutio secundaria nobis, quam Romani gramaticam vocaverunt. Hanc quidem secundariam Greci habent et alii, sed non omnes: ad habitum vero huius pauci perveniunt, quia non nisi per spatium temporis et studii assiduitatem regulamur et doctrinamur in illa. Harum quoque duarum nobilior est vulgaris: tum quia prima fuit humano generi usitata; tum quia totus orbis ipsa perfruitur, licet in diversas prolationes et vocabula sit divisa; tum quia naturalis est nobis, cum illa potius artificialis existat. Et de hac nobiliori nostra est intentio pertractare.

Provo a tradurre (ovviamente in più punti la traduzione richiederebbe non brevi giustificazioni): «Parlata popolare chiamiamo quella cui i piccoli, mentre non sanno parlare, sono abituati da chi gli sta intorno quando dapprima cominciano a distinguere le voci, ossia, per dirlo più brevemente, definiamo popolare quella parlata che riceviamo senza badare a norme imitando la donna che ci nutre. Esiste poi anche un'altra parlata secondaria, che i Romani chiamarono grammatica. Questa parlata secondaria hanno anche i Greci e altri, ma non tutti: al possesso di questa pochi pervengono, perché solo attraverso un certo spazio di tempo e abitudine allo studio impariamo a regolarci e ci addottriniamo in essa. Di queste due, dunque, la più nobile è la popolare: sia perché fu la prima consueta al genere umano, sia perché di essa fruisce tutto il mondo, quantunque sia divisa in diversi modi di proferire e vocaboli, sia infine perché a noi è naturale, mentre l'altra sussiste piuttosto in quanto artefatta. È nostra intenzione trattare qui di questa più nobile».

Non sono molti i testi in cui, da parte di persone che conoscono bene il valore della locutio secundaria, oggi diremmo dell'uso formale delle lingue scritte di cultura, e che, magari, ad essa hanno dedicato una vita di studi e di creazione, si affermi con pari nettezza il primato della locutio vulgaris. Altri testi 
consonanti e sempre più riccamente argomentati, da Leibniz, a Wilhelm von Humboldt e Søren Kierkegård, a Saussure, a Wittgenstein, a Orwell, a Italo Calvino, mi è accaduto altrove di ricordare, ma non sono molti. Il cuore dell'intellettuale istruito batte per le locutiones secundariae, in cui ha investito tempo e fatiche, e non sempre lo soccorrono la mente e il suo stesso sapere a ricordargli che senza le umili primae non vi sarebbero le amate secundariae. L'affermazione non era leggera per Dante. Era stato dapprima convinto, ai tempi della Vita Nova, che in "volgare» potessero e dovessero trattarsi solo passioni d'amore e che al resto dovesse provvedersi con la lingua grammaticale, il latino. Poi aveva alquanto cambiato idea: nel Convivio anche i volgari sono ammessi a trattare questioni intellettualmente gravi e rilevanti, ma, come leggiamo nel quinto capitolo del libro primo, permane nella sua mente il primato del latino, che supera il volgare "per nobiltà, perché lo latino è perpetuo e non corruttibile, e lo volgare è non stabile e corruttibile». Il De vulgari eloquentia è un passo deciso in una nuova direzione: la parlata popolare, volgare, è nobilior perché è la prima che si offre a ciascun essere umano e, diversamente dalle lingue filtrate dalla normazione grammaticale, è presente dappertutto per una necessità che accomuna tutti gli umani e li fa diversi da altri animali e dalle intelligenze celesti. Come si sa, questo fu solo un primo passo. Il De vulgari restò ancora pervaso dall'idea che vi fosse una gerarchia di lingue, più e meno dotate di pregio, più e meno illustri, e che tra le pregevoli e illustri non ci fosse, tra altre, proprio la sua locutio vulgaris, il fiorentino. Un altro passo decisivo verso l'affermazione della piena e pari dignità, della continuità e della storica mutevolezza di tutte le locutiones primarie e secondarie, nessuna esclusa, Dante lo avrebbe compiuto poi attraverso l'esperienza della Commedia. Ma qui per ora questo non interessa. Il filo del nostro discorso è interessato dal richiamo alla percezione dantesca del fatto che in molti casi alle locutiones native e spontanee, primarie e nobiliores, si accompagnano e sovrappongono locutiones secundariae, artificiali, che si acquisiscono non nisi per spatium temporis et studii assiduitatem.

Come oggi diremmo, a ogni locutore umano il repertorio di forme linguistiche di cui dispone offre la possibilità di muoversi nello spazio linguistico e culturale lungo tre assi. Questi hanno all'origine la immediatezza espressiva (la locutio prima) sia più accentuatamente locale, anzi privata e talora perfino familiare e personale, sia più accentuatamente informale, bisognosa, nel realizzarsi e per essere intesa, dell'integrazione con fattori esterni alla forma del segno linguistico (il contesto situazionale, le modalità semiotiche di contorno, il modo stesso delle realizzazioni foniche o grafiche dei significanti ${ }^{2}$ ecc.), sia più accentuatamente naturalis come la realizzazione vocale e percezione uditiva. Da questa origine lungo i tre assi il locutore impara, per spatium temporis et studii assiduitatem, che può spostarsi e si sposta nell'uso del repertorio

2. Su questo punto rinvio alle acute considerazioni di una valente out-sider dei nostri studi accademici, Annamaria TESTA, Le vie del senso. Come dire cose opposte con le stesse parole, Roma: Carocci, 2004. 
di cui dispone verso forme sia sempre meno locali e private, sempre più pubbliche e generalmente apprezzabili, sia sempre meno informali e sempre più, invece, tali da essere realizzate e fruite qua tales, indipendentemente (o sempre meno dipendentemente) dal contesto esterno al segno, sia, infine, sempre meno immediatamente naturali, come nella vocalità più scandita e sorvegliata, nelle scritture chirografe o a stampa, nelle altre sorte di realizzazione e trasmissione artificiale dei segni.

Ciò che abbiamo descritto è il primo dei due modi secondo cui si atteggia il plurilinguismo: è il plurilinguismo come pluralità di norme, nel senso che Luis Hjelmslev e poi Eugenio Coseriu hanno dato a questo termine, cioè come pluralità di modalità secondo cui una stessa lingua può essere «saturata» nelle sue realizzazioni. $\grave{E}$, dunque, il plurilinguismo come variabilità interna a ciascuna lingua, una variabilità costitutiva e fisiologica, da cui germina, attraverso il tempo e le vicende storiche, l'altro plurilinguismo che occorre ricordare.

$\mathrm{Vi}$ è infatti un secondo modo con cui il plurilinguismo si manifesta: non pluralità di norme, ma diversità e pluralità di differenti lingue. Ovviamente il riconoscimento dell'esistenza di una pluralità di lingue affiora da tempi remoti in culture diverse. $\mathrm{Ci}$ interessa qui ricordare che si è accompagnato ad atteggiamenti valutativi altresì diversi fin dalle origini a noi note. La pluralità linguistica ebbe il connotato di una punizione celeste, di una vera e propria maledizione, nella narrazione biblica della Torre di Babele. Al contrario apparve una benedizione nell'ancor più antico inno egiziano al Sole, e il connotato positivo e sacrale si ripropose con la narrazione della Pentecoste negli Actus Apostolorum. La Pentecoste, il divino donum linguarum, ha ispirato momenti importanti della storia delle tradizioni cristiane ed è stato all'origine di quelle molte traduzioni delle Scritture che in molti casi sono state e sono alla radice di molte attuali tradizioni linguistiche consolidate. Il riconoscimento attivo e il sostegno al plurilinguismo come pluralità di lingue interferisce con il plurilinguismo delle locutiones d'una stessa lingua. Tra le 6800 lingue che oggi contiamo nel mondo circa trent'anni fa soltanto settecento conoscevano un uso oltre che parlato anche scritto. Oggi le lingue anche scritte (ciò segnala una rilevante controtendenza rispetto a quanto credono i lamentatori della morte delle lingue, lamentatori che fanno assai notizia nei media) sono diventate 2400. Ciò si deve in una parte cospicua all'attività traduttoria delle chiese cristiane: in Europa e nel mondo lo spirito della Pentecoste ha animato una incessante attenzione delle confessioni cristiane e della Chiesa di Roma per le diversità linguistiche e si è concretato nel loro rispetto e sostegno, anche là dove le vicende politiche o della cultura intellettuale spingevano nell'opposta direzione della disattenzione, dello spregio, dell'aperto rifiuto, talora persecutorio fino alla glottofagia e all'etnocidio, delle lingue diverse e dei loro parlanti.

Anche il quadro delle riflessioni filosofiche e teoriche e delle ricerche linguistiche scientificamente fondate sul plurilinguismo è venato di contrasti. Certamente, in ambito scientifico, nessuno nega il fatto del plurilinguismo, la realtà delle 6.800 lingue diverse oggi censite nel mondo. Ma, dalle antiche filosofie greche alla linguistica moderna, il variare degli atteggiamenti di fondo 
si è tradotto nel ruolo diverso che all'evidenza della pluralità linguistica viene assegnato. Vi sono state, nell'antico mondo greco e romano come nella linguistica moderna, elaborazioni teoriche che, pur senza negare l'evidenza, si sono orientate piuttosto verso il cercare di cogliere ciò che di universalmente umano soggiace alla molteplicità linguistica. Questa, in vario modo, è stata lasciata in ombra. Come ha detto una volta ironicamente un grande linguista francese del Novecento, André Martinet, vi sono linguisti che considerano la diversità delle lingue come un fastidioso malanno professionale. Altri invece, già nel mondo antico e medievale e poi, con rinnovata capacità di apporti teorici e descrittivi, nel mondo moderno, hanno visto nella pluralità di lingue in cui si proietta la umana facultè du langage un dato, una evidenza, da assumere in modo primario per la comprensione teorica e storico-antropologica della realtà linguistica.

Oggi, pur con qualche accentuazione diversa, la teoria del linguaggio riconosce che, come Dante, seguendo Orazio e con geniale colpo d'ala, seppe scorgere e dire nella Commedia, ${ }^{3}$ l'innovatività permanente percorre l'intera realtà linguistica. Stanti le imprevedibili esigenze adattive e creative che caratterizzano la specie umana, le lingue, tutte le lingue, tutti gli insiemi di parole e loro regole d'uso, appaiono fatte per consentirla. L'innovatività permanente è una necessità vitale nel funzionamento delle lingue. Come intuì Dante, essa è per tutte la matrice del loro perpetuo differenziarsi nel tempo e nello spazio. Vi è un nesso profondo tra la varietà delle lingue e la variabilità interna a ciascuna lingua. Anche la diversità e molteplicità delle lingue non appartiene alla patologia, ma alla fisiologia del linguaggio.

Già altre volte a chi pensa di poter mettere tra parentesi la pluralità linguistica o la ritiene un fatto marginale ho proposto un semplice calcolo. I paesi che hanno un seggio alle Nazioni Unite sono circa 200, le lingue parlate sono 6800: ciò significa che mediamente ogni paese del mondo ha in sé 34 idiomi diversi. E se ritorniamo al dato prima ricordato delle lingue anche scritte, delle 34 circa 12 sono, oltre che parlate, anche scritte.

2.

Torniamo all'Italia. Le lingue ivi presenti come lingue native, di antico insediamento e di più o meno rilevante tradizione scritta, sono numerose. Anzitutto viene, per ragioni storiche e, ormai, demografiche, la lingua che dal Cinquecento si dice italiana e che oggi, dopo secoli di vita minoritaria, è nota e in uso per il 95\% della popolazione. Poi vengono i numerosi idiomi dialettali italoromanzi, articolati in almeno una quindicina di grandi raggruppamenti dialettali: piemontese, lombardo, veneto-giuliano, ligure, emiliano-romagnolo, marchigiano, toscano, umbro-aretino-chianaiolo,

3. Mi permetto di rinviare a un mio lavoro, «Porci in Paradiso. Un motivo epicureo in Dante», in L'occhio e la memoria. Miscellanea di studi in onore di Natale Tedesco, Palermo et alibi: Sciascia et alii, 2004, p. 59-70. 
abruzzese-molisano, romanesco, napoletano, pugliese, salentino, lucano, calabrese, siciliano. I trattini che si adoperano qua e là nelle denominazioni unitarie fanno affiorare, pur in forma schematica, quel che i locutori dialettali ben sanno: entro ciascun raggruppamento, in parte creato in re dal prestigio e dall'attrazione di una delle molte capitali italiane (Torino e Genova, Milano, Venezia, Bologna, Roma, Napoli, Palermo, Catania), in parte costruito dai linguisti, esistono differenze che spesso sono assai profonde e possono bloccare la intercomprensione, come avviene tra chi parla uno stretto dialetto lombardo valtellinese e chi un lombardo bergamasco, tra chi parla calabrese settentrionale e chi meridionale, chi romanesco di città e chi artenese o ciociaro. Ma qui, in questa sommaria rivisitazione d'insieme, più conta che molte parlate dialettali spiccano per un prestigio sociale irradiato dalle antiche e ancor differenti capitali, e per una tradizione letteraria e teatrale rinverdita dal cinema e dalla comicità televisiva. Con maggiore o minor fortuna, questi dialetti almeno in ricezione (ma non solo) si impongono oltre i confini nativi come è avvenuto e avviene per piemontese, milanese, genovese, veneziano, bolognese, romagnolo, fiorentino vernacolare, romanesco, ciociaro, napoletano, barese, siciliano. A questi idiomi nativi si aggiungono, come parte integrante del patrimonio linguistico nazionale, le parlate non italo-romanze che dal 1948 la Costituzione della Repubblica intendeva tutelare e che sono state enumerate e normate nel riconoscimento, anche se in modo non felicissimo, da una legge del 1999: provenzale o occitanico, franco-provenzale, francese, tedesco valser e sud-tirolese/alto-atesino, sloveno, ladino di Bolzano e di Trento, friulano, serbocroato, albanese, neogreco, catalano, sardo. Tra i suoi più evidenti difetti la legge del 1999 ha anche l'omissione delle parlate rom, presenti in Italia fin dal Cinquecento. E si è da più parti lamentata anche l'assenza di riconoscimento a quelle enclaves che, italoromanze al pari di tanti dialetti, si sono venute a creare e si trovano in aree lontane dalle originarie e affini, come il ligure a Carloforte in area sarda o i dialetti galloitalici in Sicilia.

In riferimento agli anni più recenti, il quadro del plurilinguismo di lingua va completato almeno con l'evocazione delle lingue di minoranza di nuovo insediamento, dal tagalog al somalo, all'arabo magrebino.

In modo implicito, accennando al vario uso dei dialetti, si è già detto che il plurilinguismo di lingua si intreccia con il plurilinguismo di norma. Anche se non sempre i fatti sono studiati accuratamente, possiamo postulare che per ciascuno degli idiomi ricordati sopra esistano norme di realizzazione diverse cui i locutori anche meno scaltriti sono sensibili: norme che smunicipalizzano una parlata locale per renderla comprensibile ai contermini e norme che invece accentuano la divergenza, norme che aderiscono a uno standard illustre, ove questo esista, e norme che divergono verso varianti basse, norme conformi a ideali o usi effettivi scolastici, scritti, nazionali, e norme più aderenti alle esigenze dell'informalità colloquiale. Le grandi città e antiche capitali, Firenze compresa, si offrono in modo più evidente e di facile accesso all'osservazione di questo intreccio di norme e di idiomi diversi. Ma l'intero paese è linguisticamente fatto in modo da presentarsi come un prezioso laboratorio 
di analisi di sociolinguistica interna ed esterna, solo che si vincano i pregiudizi monodisciplinari e le residue avversioni al riconoscimento della realtà del plurilinguismo nelle sue diverse modalità.

\section{3.}

Nel mondo, le scuole, da quando e dove hanno cominciato a esistere, le amministrazioni pubbliche, la politica, le esigenze di commercio ed economia hanno pesato e ancora pesano spesso nell'ottundere o reprimere anche la semplice percezione di queste differenziazioni di lingue e norme e della variabilità continua e incessante che vi si connette. E per lunghi tratti queste forze e istituzioni hanno operato per stabilizzare soltanto alcuni tra i molti idiomi reali pullulanti in ciascuna comunità privilegiando nelle attenzioni e spingendo a privilegiare in re soltanto lingue di tradizione scritta, a loro volta fissate, per il possibile, non nella loro più estesa varietà, ma secondo canoni normativi restrittivi.

Da millenni accade che queste forze si proiettino nell'assumere una sola varietà di una lingua tra le altre come la lingua e consacrarla nel e con l'uso scritto, relativamente più stabile e durevole. Ancora oggi in India due milioni circa di persone scrivono, leggono e possono tra loro parlare il sanscrito nelle forme "artefatte» (tale è, come si sa, il senso inziale della parola sanscrito) che questa lingua assunse nel IV secolo avanti Cristo. Solo da pochi anni la Chiesa di Roma ha abbandonato l'uso liturgico, ma non quello ufficiale del latino nelle forme scritte che questa lingua assunse intorno al IV secolo dopo Cristo. Un grande studioso, Edward Said, ha dato di recente, in quello che è stato purtroppo suo ultimo contributo, un quadro assai suggestivo delle ragioni di persistenza dell'arabo classico scritto nel variegato mondo arabofono. Nella diacronia sono casi estremi di quel sovrapporsi stabile degli usi scritti alla incessante variabilità delle parlate, casi estremi di quella garanzia di convergenza, incurante o poco curante delle variazioni del parlato, che le lingue scritte offrono attraverso il tempo e lo spazio a chi le usa. Questa garanzia è stata apprezzata in Europa dagli Stati nazionali formatisi a partire dalla tarda età medievale fino al Novecento: per ciascuno di essi si è vagheggiato e si è cercato dalle classi dirigenti che vi fosse un'unica lingua, espressione dell'unità e indipendenza della nazione e dello Stato e garanzia sostanziale (questa una sua forza indubbia) della parità dei sudditi, poi dei cittadini dinanzi alla legge. Tra Cinquecento e la metà del Novecento soltanto in alcune aree atipiche, come la Confederazione elvetica, l'Impero absburgico e, dopo la Rivoluzione d'Ottobre, l'Unione Sovietica, si è formalmente ammesso che entro gli stessi confini potessero coesistere più lingue di pari dignità. E soltanto nella seconda metà del Novecento questa ammissione formale ha guadagnato terreno tendendo a generalizzarsi entro gli Stati dell'Unione Europea.

Il privilegio accordato alla norma scritta e meno informale di un idioma preferito tra gli altri di una stessa comunità ha creato in tutte le aree del mondo $\mathrm{e}$ in Europa una continua dialettica tra l'adesione conservativa a una norma e 
una lingua assunte a superiori e le altre norme e lingue respinte a rango più basso, de facto coesistenti, de facto più o meno incidenti nell'uso stesso delle norme e lingue superiori, ufficiali, nazionali. Come riconobbe chiaramente Saussure nel terzo dei suoi corsi di linguistica generale, nessun aspetto di questa incessante dialettica tra conservatività e innovazione, tra diversificazione $\mathrm{e}$ convergenza va trascurato e nessuno va mitizzato o demonizzato in nessuna sede, negli studi scientifici come nella progettazione e pratica scolastica. Ai lodatori estremisti dell'uso parlato come unica realtà cui guardare occorre ricordare che un loro progenitore, il sommo Platone, ha affidato nel Fedro ad alcune pagine scritte tra le più alte della Weltliteratur (in ciò vi fu certo una buona componente di impassibile ironia) il suo elogio dei lógoi ágraphoi, del linguaggio non scritto. E a chi crede di potere chiudere le porte alle innovazioni rampollanti nel parlato si potrà ricordare che a queste, alle "parole che sono sulla bocca di tutti», hanno guardato e attinto i grandi creatori e prosecutori delle tradizioni letterarie, da Dante a Leopardi o a Montale e Gadda, da Rabelais a Raymond Queneau, da Lutero a Karl Kraus, ai traduttori e ritraduttori dei testi biblici ed evangelici. E, comunque, osservava Saussure nelle lezioni del terzo corso, per ogni lingua scritta che riesca a isolarsi davvero dal fluire del parlato viene il tempo del suo crollo repentino, come di una crosta glaciale che si dissolve nell'impeto delle correnti soggiacenti. Ma, di nuovo, lo spettacolo della storia linguistica non deve far dimenticare quanto, morendo, una tradizione linguistica scritta sa e può dare ad altre: quanto il cinese scritto ha dato all'affrancantesi giapponese, quanto il sanscrito ha dato alle lingue dell'India medievale e moderna e, attraverso la colonizzazione religiosa buddista, a lingue dell'Estremo Oriente, quanto il patrimonio scritto greco e latino classico ha dato e dà ancora alle lingue dell'Europa moderna.

Attraverso la sempre più diffusa istituzione di scuole e la creazione di amministrazioni pubbliche statali, le tendenze che hanno spinto a isolare e privilegiare solo una norma tra le possibili di una lingua e solo una lingua tra le molte in presenza, hanno operato in Europa da più tempo e più largamente che altrove. Queste tendenze, come ho ricordato, hanno operato in Europa già dall'antichità e dal Medio evo e con forza ancor maggiore, forza impositiva, forza trascinante, ancor più nell'Europa moderna. E tuttavia la pluralità linguistica è talmente radicata entro la più profonda natura stessa del linguaggio che essa ha resistito: ha resistito come pluralità di norme per ciascun idioma, ha resistito come pluralità di idiomi. Perfino se si bada alle sole lingue riconosciute ufficialmente dagli Stati dell'Europa contemporanea, constatiamo che su 48 Stati dell'Europa «dall'Atlantico agli Urali» le lingue ufficialmente riconosciute sono 53. Per paradosso perfino il costituirsi di Stati nazionali indipendenti, con la conseguente spinta a elevare una lingua a rango di lingua nazionale e ufficiale, ha contribuito alla accentuata fisionomia plurilingue dell'Europa d'oggi. Se poi osservassimo l'intera effettiva realtà dei parlati nativi, l'Europa, come fu rilevato già da Albert Dauzat e come è possibile constatare perfino ai linguisti se si tolgono gli occhiali oscuranti dell'ideologia monolinguistica, apparirebbe morcelèe, frantumata, come è l'area bantu o come furono le aree 
amerindiane, in centinaia e centinaia di idiomi diversi. In quello spazio in cui la politica e la legislazione contano una lingua soltanto (l'italiano o lo spagnolo o il catalano o il francese o il friulano), là dove lo stesso linguista o dialettologo per esigenze di classificazione conta solo uno dei grandi raggruppamenti dialettali (il "piemontese» o il «napoletano», il «basso tedesco», il «bavarese» o l'«alemanno» ecc.), le comunità di parlanti nella loro quotidianità reale si raccolgono e dividono secondo limiti assai più fittamente disposti e quindi ben più numerosi.

Il plurilinguismo italiano ha una dimensione più accentuata, particolare, rispetto ad altre aree europee. Ma il linguista e anche lo storico della lingua italiana devono sapere che esso non è un lusus naturae o un'invenzione ideologica o un "pregiudizio storiografico", come qualcuno si è spinto a dire, ma è una corposa realtà saldamente radicata nell'intera storia europea e, occorre dirlo?, nella storia stessa dei popoli e delle culture intellettuali e letterarie dell'Italia antica, medievale e moderna.

4.

Torniamo a guardare all'insieme del paesaggio linguistico italiano d'oggi. Lo abbiamo considerato nei paragrafi precedenti in modo relativamente statico e nella sua superficie: la lingua italiana da un canto, con le sue appena evocate norme di realizzazione; le lingue non italoromanze meno diffuse dall'altro, anch'esse con le solo evocate varie norme di realizzazione; i dialetti italoromanzi, sulle cui varietà interne abbiamo speso qualche parola in più; le lingue minoritarie di nuovo insediamento. Vorrei ora accennare ad alcune dinamiche che investono nel complesso la comunità dei locutori italiani e, se posso insistere nel paragone geografico, ad alcune faglie che soggiacciono al paesaggio statico evidente e che sono sotterraneamente in movimento.

Le tendenze dinamiche più evidenti paiono due: la sempre più larga base di locutori che nell'uso convergono sempre più spesso verso l'italiano; la crescente accettazione dell'esistenza e della utilizzabilità di distinte norme di realizzazione non solo dell'italiano, ma di tutti i numerosi idiomi in presenza.

Può darsi che nel 1861 coloro che parlavano attivamente italiano fossero più del 2,5\% (come a me parve di dover computare quarant'anni fa) e che coloro che capivano discorsi e testi in italiano arrivassero a sfiorare quasi il $10 \%$, come ha sostenuto anni fa il compianto Arrigo Castellani: comunque per nove su dieci l'italiano era allora una realtà estranea. Per motivi che sfuggono, la discussione si è concentrata sul dato del 1861 e (che io sappia) non è stato invece contestato il dato degli anni cinquanta del Novecento. Allora, cinquanta anni fa, secondo una stima che volutamente massimizzava gli indicatori della pratica dell'italofonia, si ipotizzò che l'italofonia attiva e abituale fosse propria del $18 \%$ della popolazione, che un altro $18 \%$ potesse alternare all'uso d'uno dei dialetti l'uso dell'italiano, e che per il restante $64 \%$ gli idiomi disponibili fossero soltanto i dialetti italoromanzi e le allora misconosciute lingue di minoranza, tra le quali le due di maggior rilievo demografico (le 
parlate sarde e friulane) nella comune opinione venivano annoverate tra i dialetti italoromanzi. Questo dato era congruo ai dati del 1861 e soprattutto si è rivelato congruo ai dati che si sono accumulati negli anni seguenti grazie ad altri sondaggi e stime e poi grazie alle rilevazioni campionarie delle autovalutazioni promosse dalla DOXA e dalle accurate indagini multiscopo dell'Istat. In base a queste si è potuto vedere crescere se non quasi di anno in anno, di quinquennio in quinquennio il numero di coloro che dichiaravano di usare sempre e solo o in modo prevalente l'italiano in ogni occasione e si è visto diminuire il numero di coloro che usano in modo esclusivo sempre e solo uno dei dialetti. Un dato significativo è che tra i due estremi si è formato ed è cresciuto quello che altrove ho chiamato il partito (linguistico) di maggioranza relativa (anzi a vero dire assoluta): la grande schiera di coloro che usano alternativamente, a seconda del bisogno e delle circostanze, ora l'italiano ora una delle altre parlate esistenti in Italia. Secondo gli ultimi dati disponibili i tre gruppi si ripartiscono con le seguenti percentuali (che qui arrotondo leggermente): il $44 \%$ parla in modo esclusivo o prevalente italiano, il 5\% parla uno dei dialetti o altri idiomi, il 51\% parla alternativamente italiano o uno dei dialetti.

L'analisi particolareggiata di questi dati e del loro variare diacronico e sincrono per genere, regione, tipo di centro abitato, classi anagrafiche e socioculturali è del massimo interesse e vorrei qui augurarmi che una linea di lavoro di questa nuova rivista possa essere proprio lo sviluppo sistematico di tali analisi, di cui abbiamo già alcune testimonianze significative, ad esempio per la Sicilia, il Salento, Napoli, Torino, il Friuli. Dal moltiplicarsi integrato di tali analisi, dal costituirsi di un «intellettuale collettivo» che le cumuli e sintetizzi, possiamo sperare che entri in funzione quell'osservatorio linguistico italiano che Pier Paolo Pasolini vagheggiava e che ci dica per annos "che lingua fa" in modo il più oggettivo possibile, sottratto ai malumori di qualche dotto collega e alle sciocchezze che "fanno notizia» e "fanno titolo" nell'informazione corrente.

Qui vorrei soltanto rammentare ancora una volta che le popolazioni italiane, nella lunga loro storia che ricostruiamo e conosciamo lungo tre millenni, mai avevano sperimentato un pari grado di convergenza effettiva abituale (il 44\%) e di convergenza potenziale e spesso effettiva (il 95\%). verso uno stesso idioma. E vorrei anche sottolineare che questa convergenza si è realizzata non già scacciando e cancellando l'uso di altri idiomi, ma, secondo la linea preconizzata da Carlo Cattaneo e Graziadio Ascoli, da Francesco De Sanctis, Francesco D’Ovidio e Giuseppe Lombardo Radice, da Giacomo Devoto e dalle Dieci tesi per una educazione linguistica democratica: si è realizzata cioè cercando di salvaguardare e anzi accrescendo per ampi strati di locutori il plurilinguismo caratteristico della tradizione italiana e, come ho detto, europea. ${ }^{4}$

4. Per questo sfondo storico-teorico rinvio a Silvana FERreri, T. De MAUro, «Glottodidattica come linguistica educativa», in Grazia BASILE, Annarosa Guerriero, Miriam VogheRA (a cura di), E.Li.Ca. Educazione linguistica e conoscenza per l'accesso [all'università], Perugia: Guerra, 2004 (in stampa). 
Questo processo esterno, che senza enfasi credo si possa dire di portata storica, ha avuto e sta avendo riflessi interni sulle lingue in presenza. Il crescere della convergenza verso la capacità d'uso dell'italiano rende difficile la vita a ogni proposito di normare dall'alto, da un qualunque luogo particolare, i modi d'uso della lingua. Il liberalismo linguistico che Benedetto Croce preconizzava all'inizio del Novecento è ormai ben radicato nella coscienza di decine di milioni di locutori. Qualunque imbragatore, Grande Fratello o Piccolo Padre, avrà (avrebbe, si spera, se vorrà provarci) vita difficile. Ciò che linguisticamente è o non è accettabile viene di continuo sottomesso al vaglio dell'uso effettivo, anche contrastante, liberamente contrastante, di milioni di aventi diritto. Ma quanti sono in grado di esercitare responsabilmente, con sufficiente perizia di nativi, questo diritto? Prima di cercare una risposta a questa domanda osserviamo ancora che nelle dinamiche che si sono create nell'Italia linguistica contemporanea si possono segnalare alcune linee di tendenza dominanti:

l'accentuarsi del processo di italianizzazione interna, lessicale, morfosintatti$\mathrm{ca}$, fonologica dei dialetti e di parte almeno delle lingue di minoranza, senza che tuttavia siano scomparse potenzialità di innovazioni divergenti dall'italiano;

il completamento di un lessico standard, nazionale, della quotidianità e dell'alta disponibilità, oltre che del lessico tecnico più comune;

l'accentuarsi di una progressiva selezione dell'ipertrofia sinonimica e morfologica che caratterizzava ancora negli anni sessanta l'italiano e specialmente l'apparato tematico e desinenziale verbale e ancora lo caratterizza, ma sempre meno, una selezione che pare specialmente accentuata tra locutori colti di giovane generazione.

Se la possibilità e capacità d'uso d'una stessa lingua entro una società è un bene, nel senso che facilita e fluidifica i rapporti tra individui, gruppi, classi e li rende più sicuri e rende le persone più «eguali» (come don Lorenzo Milani appassionatamente insegnava), se è un bene che la morfologia e la sinonimia di tale lingua non offrano troppe portae geminae, troppi dubbi impaccianti, ma soluzioni condivise (magari anche solo per comprare qualcosa in un negozio o al mercato in una qualunque città), si può e deve concordare con Giulio Lepschy quando afferma che l'italiano nei secoli non ha mai goduto di altrettanta buona salute. Ciò che vale per l'italiano vale anche per gli italiani?

\section{5.}

Nel profondo della realtà linguistica e culturale del paese, nonostante evidenti motivi di accrescimento della sicurezza linguistica di chi usa l'italiano e di consolidamento dello standard, vi sono diversi motivi per cui non possiamo dire: dormite securi, cives. Il paesaggio linguistico italiano è tormentato da faglie e fratture soggiacenti di cui anche chi descrive la condizione linguistica del paese e chi opera in essa, giornalista, insegnante, politico, scrittore, deve farsi consapevole. 
La prima faglia consiste nella eccessiva disparità tra capacità di controllo del parlato e capacità di controllo, anzi di puro e semplice rapporto con lo scritto. Come si è detto, 95 italiani su cento non hanno grandi dubbi su come gestire la comunicazione parlata. Forse o senza forse l'italiano non è un popolo di navigatori e di santi, come asseriva il Mussolini, ma certo si sta rivelando un popolo di parlatori proclivi al parlare senza interruzioni. La propensione al parlato non è l'unica causa, forse, ma certo è assai rilevante per spiegare il travolgente successo che nel confronto internazionale in Italia hanno i telefoni mobili, cellulari, i telefonini (io avanzo l'ipotesi che pesi molto anche la consapevolezza dell'incertezza e internazionalmente nota inefficienza dei pubblici servizi). L'AIART, Associazione Italiana Ascoltatori Radio Telespettatori, un'associazione di ispirazione cattolica costituitasi negli anni cinquanta, ha organizzato di recente un convegno sulla telefonia mobile. Qui Cristina Torcia, responsabile della comunicazione di Vodafone, ha fornito un dato poi ripreso e commentato dalla stampa: l'Italia ha la più alta percentuale europea di telefoni cellulari attivi, che nella primavera 2004 sono arrivati a cinquanta milioni. Tolti i bambini, la media è di un cellulare a testa, la più alta oggi nel mondo. Un viaggio in treno, una passeggiata per strada o, purtroppo, una riunione o conferenza e perfino a volte un concerto dicono, più di ogni statistica, quanto i telefonini siano continuamente in funzione. Dagli studi condotti per realizzare anni fa il lessico di frequenza dell'italiano parlato sappiamo che la conversazione telefonica pare fungere da lettino dello psicanalista e liberare senza remore l'uso più informale del repertorio linguistico di cui si dispone.

Tutt'altri i dati sulla lettura di giornali e di libri. La lettura di giornali è restata percentualmente immobile dagli anni cinquanta del Novecento a oggi: contro medie europee di un quotidiano venduto per ogni due abitanti, in Italia si vendevano mezzo secolo fa e si vendono oggi un quotidiano ogni dieci abitanti. Se si accetta la stima per cui una copia di quotidiano ha in media tre "percettori», solo un terzo della popolazione italiana è raggiunto in un giorno dalla lettura di quotidiani. Quanto ai libri, gli indicatori sono migliorati sensibilmente negli ultimi decenni per le classi giovani e per le donne, ma anche la lettura di libri non va oltre un terzo della popolazione, secondo un dato che forse nasconde non poche risposte di prestigio.. Un dato sicuro che l'Istat ha accertato ci dice che soltanto il 10\% delle famiglie italiane spende nell'anno qualche euro per libri non scolastici. Né le cose vanno troppo meglio per i redditi più elevati. Ai livelli di più alto reddito qualche spesa per libri non scolastici è fatta soltanto dal $19 \%$ delle famiglie. La lettura si configura come una faccenda puramente scolastica. Si aggiunga che, interrogati dall'Istat sulle motivazioni della non lettura, i non leggenti per il $6 \%$ rispondono con un candido e onesto «non so leggere».

Tra le pieghe delle tabelle Istat emerge la vasta, oscura schiera degli analfabeti. La persona istruita stenta a credere alla loro esistenza. E non senza ragione perché gli analfabeti hanno sviluppato, come parte integrante delle loro tecniche di sopravvivenza, una grande astuzia per nascondere la loro condizione. In mancanza di ricerche empiriche su individui, la letteratura ci dà per 
ora il meglio. Nella Macchia umana di Philip Roth c'è un personaggio complesso, una donna, che si finge analfabeta e si isola dal mondo, scegliendo di svolgere solo lavori elementari. In un bellissimo pseudogiallo di Ruth Rendell, un giallo in cui dall'inizio si conosce l'assassino e perfino il movente, ma non il groviglio psicologico, Judgment in Stone, tradotto in italiano come La morte non sa leggere (Claude Chabrol ne ha tratto sullo schermo Il buio nella mente), di nuovo protagonista è una persona analfabeta (de facto, pur avendo completato le scuole dell'obbligo!), che, scoperta, stermina la famiglia presso cui era a servizio. In Germania, un magistrato, Bernhard Schlink, è diventato un bestseller con lo stesso tema che sorregge un altro giallo-non giallo, $A$ voce alta. Nella serie televisiva tedesca nota in Italia come "Il Commissario Rex», nella puntata Una dritta mortale (RAI1 del 30.4.2004) un analfabeta, vergognoso della sua condizione e di frequentare un corso per adulti per uscirne, non dice la verità su quel che fa in una certa ora della sera (frequentando il corso) e rischia di farsi condannare perché privo di alibi per un assassinio commesso in realtà da un suo "colto» amico cui aveva confidato l'esistenza di gioielli in casa d'un ricco gioielliere a riposo. La verità però viene fuori, l'analfabeta si salva e il colto amico, inseguito dalla polizia, pur sapendo leggere, non legge un cartello di pericolo, cade in un baratro e muore. ${ }^{5}$

Questo ci porta in pieno all'interno della seconda faglia: la linea di frattura tra capacità di parola parlata e capacità di rapporto produttivo o ricettivo con la parola scritta. Chi mostra grandi preoccupazioni per la sorte della lingua italiana dovrebbe aver prestato e prestare attenzione ai dati che qui ancora richiamo sperando in un soprassalto d'attenzione. Nel 2000 si è conclusa una seconda indagine comparativa internazionale, SIALS, Second International Aldult Literacy Survey, condotta in Italia dal Centro Europeo dell'Educazione e svoltasi, attraverso la somministrazione di cinque questionari graduati a campioni di popolazione tra i 16 e i 65 anni in 22 paesi. I questionari miravano ad accertare a livelli crescenti, dal più elementare al più complesso, la capacità di comprendere testi scritti, di produrli e di eseguire calcoli. I 22 paesi si distinguono in tre grandi gruppi: (1) paesi in cui la competenza alfabetica funzionale dei livelli più alti (superamento dei questionari 3, 4 e 5 della scala Sials) appartiene a più del $50 \%$ della popolazione, e cioè oltre metà della popolazione è in possesso di strumenti solidi e durevoli di lettura, scrittura e calcolo, capaci di arricchirsi ulteriormente: Germania 51,4\%, Belgio 52,3\%, USA 53,5\%, Nuova Zelanda 54,2\%, Danimarca 54\%, Australia 55,8\%, Canada 57,8\%, Paesi Bassi 59,4\%, Finlandia 63,3\%, Norvegia 66,8\% e Svezia 72,1\%; (2) paesi in cui i livelli 3, 4 e 5 sono raggiunti da più del $40 \%$ della popolazione: Svizzera 46,2\%, Repubblica Ceca 46,2\%, Irlanda 47,6\%, Regno Unito

5. Sul tema dell'analfabetismo nella letteratura cfr. Julia GENZ, Alphabeten und der blinde Fleck der Literatur, München: Wilhelm Fink Verlag, 2004, e, in altra prospettiva, Raffaella PETRILLI, Il detective e le parole. Le strutture semantiche del giallo, Troina (Enna): Città Aperta Edizioni, 2004, in particolare p. 46 e s., e Massimo Vedovelli (a cura di), Lingua in giallo, Perugia: Guerra, 2004. 
47,9\%; (3) paesi in cui la quota di popolazione che riesce a raggiungere questi livelli è inferiore al 35\%: Cile 14,9\%, Polonia 22,9\%, Portogallo 22,9\%, Slovenia 23,3\%,Ungheria 23, 4\%, Italia 34,5\%.

Come si vede, l'indagine osservativa ci dice che solo un terzo della popolazione italiana è in grado di leggere un libro senza troppa fatica, di redigere un testo scritto, di eseguire o comprendere un calcolo. Ma non basta. Dall'indagine risultano anche altri due dati: il $5 \%$ della popolazione non è in grado di accedere nemmeno al primo questionario, non lo sa decifrare, è, cioè, interamente analfabeta; e il 33\% risponde al primo questionario, assai elementare, ma non riesce a rispondere alle domande del secondo, appena più complesso, e viene definito semianalfabeta. In un modo o nell'altro più del $65 \%$ della popolazione non ha un sufficiente rapporto né con lettura e scrittura né con numeri e calcoli.

C'è una frattura, dunque, celata sotto la diffusa capacità di usare l'italiano nel parlare: il 95\% della popolazione dichiara di saper parlare italiano, ma solo una parte (fra il 30 e il 50\%) dichiara di avere rapporto con la lettura di libri e giornali e solo una parte assai più ristretta, quella che accede ai questionari di livello $3,4,5$, mostra di possedere un buon possesso delle capacità di lettura e scrittura.

Per l'uso pienamente competente di una lingua come l'italiana, di antica tradizione, con una forte presenza di lessemi e strutture morfoderivazionali del latino e ancora ricca di scelte poco standardizzate, e in una comunità tanto caratteristicamente plurilingue per il coesistere di idiomi diversi e, per ciascun idioma, compreso l'italiano, per il coesistere di norme d'uso diverse, la mancanza di un esteso rapporto dei locutori con la tradizione scritta è un ostacolo malamente superabile per arrivare a un uso pienamente controllato della comprensione e della produzione linguistica. Questa linea di frattura tra capacità d'uso dell'italiano e capacità di lettura e scrittura rinvia alle condizioni in cui è avvenuto il grande aumento dei livelli di scolarità dagli anni cinquanta del Novecento ai nostri giorni. Un dato che il ceto intellettuale e dirigente cerca di rimuovere dalla memoria è che mezzo secolo fa il 59,2\% della popolazione di oltre 14 anni era privo di ogni titolo scolastico, anche la semplice licenza elementare. Non è una stima, rammento, è un dato stabilito dall'Istat sul totale della popolazione. Se si ignora questo dato, come purtroppo spesso avviene, non si è in grado di intendere che la scuola italiana nei decenni di vita repubblicana e democratica ha fatto un lavoro enorme per sottrarre il paese a questa condizione di descolarizzazione forzata, retaggio di secoli di arretratezza. Già negli anni novanta quei quasi due terzi di senza scuola degli anni cinquanta erano ridotti a pochi punti percentuali. Lo dobbiamo alla scuola. E dobbiamo alla scuola se le fasce giovani della popolazione hanno conquistato livelli di scolarità tre volte superiori a quelli delle generazioni anziane. I figli e nipoti dei senza scuola di ieri hanno conquistato tutti fin dagli anni ottanta la licenza elementare, quasi tutti negli anni novanta la licenza media dell'obbligo e nei primi anni duemila giungono per il $75 \%$ a conquistare il diploma negli esami di stato al termine delle scuole secondarie. 
Un non secondario riflesso della accresciuta scolarità giovanile deve leggersi nei recenti dati Istat secondo i quali specialmente tra i giovani sotto i 25 anni si va sviluppando una qualche familiarità con le lingue straniere, inglese, francese, poi, in misura purtroppo assai più modesta, spagnolo e tedesco e infine, in percentuali da enzima, russo e arabo. Troppo poco per le esigenze di una società europea moderna. Troppo poco specie se entro la familiarità si ricercano i dati relativi a una buona comprensione e produzione del parlato e a una buona competenza dell'uso scritto. Anche questi dati dai primi anni novanta al 2000 appaiono in crescita, ma non si va per ora oltre il quinto della popolazione. Tre quinti sono ancora solo orecchianti di una lingua straniera. Un altro quinto non giunge neanche a questo. Il lavoro da fare resta enorme. Ma la generazione più giovane ha una funzione guida anche in questo processo, decisivo per la buona salute linguistica di un paese europeo moderno.

I progressi compiuti nei livelli di scolarizzazione sono stati dunque enormi e tuttavia vanno segnalati due grandi limiti. Le eredità del passato e la mancanza di un sistema di educazione degli adulti fanno sì che ancora nel 2000 quasi il 50\% della popolazione adulta sia privo di licenza media dell'obbligo o titoli superiori. Per metà della popolazione la licenza elementare è il tetto dell'istruzione. In una società contadina, statica, raggiungere questo tetto dava ai privilegiati che vi arrivavano un sufficiente grado di controllo del loro mondo culturale e degli stessi idiomi in presenza. Oggi, in una società postindustriale, mobile, aver raggiunto soltanto quel tetto è del tutto insufficiente.

Si aggiunga inoltre che indagini comparative internazionali sempre più accurate ci dicono che in ogni paese la qualità culturale dell'ambiente familiare si riflette in generale sulla qualità degli apprendimenti scolastici. L'Italia nel complesso non fa eccezione. I figli di quei due terzi di popolazione che attualmente non sono andati oltre la licenza media, che hanno un cattivo o assente rapporto con la lettura, grazie all'impegno loro e delle scuole possono ben procedere verso i diplomi e le università, ma a prezzo di promozioni che in molta parte sono più formali che sostanziali. Come la manchevole istruzione formale tra gli adulti, la manchevole qualità sostanziale dell'istruzione tra una metà circa delle giovani leve di diplomati porta a un rapporto problematico con l'uso della lingua italiana.

Sotto la superficie della ormai larga convergenza verso una stessa lingua, si celano dunque linee di frattura profonde che non passano più attraverso differenze regionali o di reddito, ma attraverso forti disparità nel rapporto con lettura e scrittura, nella stessa capacità potenziale di accesso a testi scritti e alla loro redazione e di calcoli, nella disparità di apprendimenti anche a parità di livelli formali di istruzione.

Chi sa, evangelicamente, che le lingue sono fatte per gli uomini e non gli uomini per le lingue, e sa, orazianamente, che mortalia facta peribunt, nedum sermonis stet honos et gratia vivax, è portato coerentemente a preoccuparsi, più che di singole sciatterie linguistiche, delle difficoltà dei parlanti e, quindi, di queste fratture nella comunità italiana che impacciano per molti l'uso pienamente competente e per tutti l'eguaglianza sostanziale (richiesta dall'art. 3, 
comma 2 della Costituzione). Ma anche chi professa amore per la lingua italiana qua talis dovrebbe intendere che essa tanto più e meglio si consolida nelle sue forme e regole quanto più si colmino e sanino le fratture che si sono qui ricordate, quanto più si rimuovano gli ostacoli che impediscono a troppi di padroneggiare con pienezza la lingua comune.

Fratture e ostacoli non sono insuperabili. Un innalzamento quantitativo e qualitativo dei livelli di istruzione delle giovani generazioni e degli adulti, la promozione della lettura e del bisogno di leggere e informarsi, lo sviluppo di stili di vita che favoriscano l'apprezzamento della cultura intellettuale, dei saperi, delle scienze: sono i maggiori percorsi che altre società non solo europee hanno imboccato e stanno seguendo per superare quegli ostacoli e quelle fratture. Pare chiaro che ciò sia possibile anche in Italia: è un impegno più lungo e faticoso di qualche predica contro questo o quel malvezzo linguistico, ma è l'unica via per migliorare realmente la condizione linguistica di tutta la popolazione. Del resto, è un impegno che sta dinanzi al paese non solo per i motivi linguistici e culturali qui in primo piano.

Nelle librerie, nelle biblioteche, nelle scuole, nei teatri, nei corsi per adulti, nel modo di fare e ricevere informazione si può e si deve combattere la buona battaglia per migliorare le condizioni linguistiche del paese. 